1

2

3

4 Monica Gomaraschi ${ }^{1}$

5

$6{ }^{1}$ Centro E. Grossi Paoletti, Dipartimento di Scienze Farmacologiche e Biomolecolari,

7 Università degli Studi di Milano, Milan, Italy

8

9

10

11

12 Corresponding author:

13 Monica Gomaraschi, PhD

14 Centro E. Grossi Paoletti, Dipartimento di Scienze Farmacologiche e Biomolecolari,

15 Università degli Studi di Milano

16 Via Balzaretti 9, 20133 Milan (Italy)

17 monica.gomaraschi@unimi.it

18

19

20

21

22 \section{DEPENDENT CANCERS}

\section{THE ROLE OF LIPOPROTEINS IN THE MICROENVIRONMENT OF HORMONE-}




\section{Abstract}

24 The tumor microenvironment (TME) is an attractive target to develop novel strategies for 25 hormone-dependent cancers. Several molecules in the TME can favor tumor development 26 and progression, including lipoproteins. Lipoproteins are taken up by cancer cells providing 27 them with cholesterol and fatty acids. Cholesterol regulates cell signaling and it is converted 28 into a series of bioactive metabolites, including hormones. The conflicting results of 29 epidemiological and interventional studies suggest that the local availability of lipoproteins in 30 the TME is more relevant for cancer biology than their circulating levels. Thus, reducing 31 lipoprotein uptake and stimulating cell cholesterol efflux to high density lipoproteins (HDL) can 32 represent a novel adjuvant strategy for cancer management. HDL-like particles can also act 33 as drug delivery systems for tumor targeting. 


\section{Relevance of the microenvironment in tumor development and progression}

35 Hormones can drive the development and the progression of malignancies at multiple sites, 36 including adrenal, thyroid, parathyroid, pancreatic, prostate and reproductive tissues. This review is mainly focused on breast and prostate cancers (BC and PCa), which are the leading cause of cancer-related death in women and men, respectively. They are classified as hormone-dependent cancers due to the key role of steroid sexual hormones in tumor initiation and progression (see Box 1). Both types of cancer are characterized by an effective response to hormone-deprivation therapies; however, therapeutic options for locally advanced or metastatic tumors are limited and poorly effective [1,2]. In the attempt to identify the mechanisms responsible for tumor progression and to find novel therapeutic targets, the tumor microenvironment (TME) has gained increased attention. The TME is as a complex, acidic and hypoxic environment, with cancer cells and non-transformed stromal cells of different origins immersed in the extracellular matrix (ECM), that evolves during tumor progression [3]. Cellular components include endothelial cells and pericytes, fibroblasts, adipocytes, resident and infiltrating immune cells, while the ECM, which provides a structural support for these surrounding cells, is mainly composed by collagen, elastin, fibronectin, laminin and proteoglycans (PGs). The role of the different stromal cells in tumor development and progression is complex and still debated, as recently reviewed by Hanahan and Mittal $[4,5]$. Interestingly, TME can modulate malignant progression in multiple ways and TME signature can predict disease outcome and therapeutic response, independent from cancer cell features, as in the case of $\mathrm{BC}[6,7]$.

Several molecules in the TME can favor the development and progression of hormonedependent cancers. It is well established the role of pro-oxidant and pro-inflammatory molecules, which can reach the TME from the systemic circulation or can be locally produced by stromal cells [8]. In addition, sources of cholesterol and fatty acids are present in the TME; these molecules can promote cancer cell proliferation through several mechanisms beyond their well-known structural and energetic role in cell physiology (see Box 2). Cholesterol and its metabolites in cancer cells

The role of cholesterol in the context of cancer cell proliferation has been recently revised. In the past, cholesterol need was ascribed to its structural role in cell membranes, while now it is recognized as a key player in the regulation of cellular function (Figure 1). Its presence in cell membranes is able to modulate the activation of transmembrane receptors [9]. In addition, it 
can be converted into a wide series of biologically active metabolites, such as oxysterols and isoprenoids [10]. Finally, cholesterol acts as a building block for the local synthesis of hormones. Consequently, cholesterol demand is higher in hormone-dependent cancers compared to other tumor types [11].

Cholesterol can be directly synthetized by cancer cells or it can be taken up by lipoproteins (see box 3 and box 4), which are present in extracellular fluids (Figure 1). Indeed, the receptor-mediated endocytosis of apoB-containing lipoproteins and, depending on the cholesterol gradient, the SR-BI-facilitated cholesterol influx from HDL, are relevant sources of cholesterol in the TME [12]. On the contrary, HDL could help in reducing cholesterol content of cancer cells acting as acceptors of cholesterol efflux [13]. To deal with their need of cholesterol, cancer cells are generally characterized by an increased expression of SREBPregulated genes, which support cholesterol synthesis and uptake, and by a concomitant inhibition of LXR-regulated genes, including $A B C$ transporters [14,15] (Figure 2). Cancer cell uptake of lipoproteins in the TME is promoted by hypoxia; indeed, in several cancer cell lines, hypoxia was shown to promote the expression of many lipoprotein receptors, as LDL-R, LRP1, VLDL-R and SR-BI [16] (see Box 3). In addition, the acidic environment increases the affinity of LDL for PGs, which favors LDL uptake by endocytosis [16]. Furthermore, PG-bound LDL are entrapped in the extracellular space, where they can undergo oxidation that increases LDL affinity to scavenger receptors and triggers pro-inflammatory and pro-oxidant cascades [17].

The relevance of cholesterol for proliferating cancer cells is supported by three main findings. First, cholesterol accumulates in cancer cells due to the upregulation of its synthesis and uptake [18-21] (Figure 2). Since unesterified cholesterol is toxic, it is quickly esterified in the cytosol by ACAT and stored in lipid droplets (LDs), which aberrantly accumulate in tumor cells [22]. Second, the inhibition of the SREBP pathway exerts antiproliferative and antimetastatic effects [20]. Third, when cholesterol is rapidly removed from cell membranes, as by methyl- $\beta$-cyclodextrin ( $\beta M C D$, an acceptor of cholesterol through passive diffusion), cell survival is impaired [23-25].

\section{Structural and functional role of cholesterol in the cell membrane} Cholesterol regulates the fluidity of the cell membrane and its distribution is not homogeneous; lipid rafts are sub-domains of the membrane particularly enriched in 
cholesterol along with glycosphingolipids and sphingomyelin [9]. Lipid rafts have a key role in signal transduction, since many types of proteins are recruited to these domains, as glycosylphosphatidylinositol-anchored proteins, prenylated and acylated proteins, and transmembrane receptors [26]. Lipid rafts act as concentrating platforms for receptors activated by ligand binding; the activation leads to rafts' clustering, which favors the interaction among the various members of a signaling complex by close proximity [26]. Interestingly, many receptors for growth factors (as epidermal growth factors, insulin-like growth factor 1 and vascular endothelial growth factor) are located in lipid rafts and an enrichment in cholesterol was shown to increase the activation of downstream PI3K/Akt and ERK1/2 pathways, likely supporting cancer cell proliferation and migration. On the contrary, when cholesterol is removed from lipid rafts (as by $\beta M C D$ or $\mathrm{HDL}$ ), receptors are internalized and signaling events blunted [24,25].

\section{Intra-tumor formation of cholesterol metabolites}

The local production of dihydrotestosterone and $17 \beta$-estradiol from cholesterol by $5 \alpha-$ reductases and aromatases could explain the progression of $\mathrm{PCa}$ and $\mathrm{BC}$ even with the very low levels of circulating hormones achieved during deprivation therapies. Locally produced hormones can activate their cognate receptors, supporting cancer cell proliferation [27,28]; accordingly, $5 \alpha$-reductase and aromatase inhibitors represent a therapeutic strategy for the management of hormone-dependent cancers [29]. Likewise, intra-tumor hormone synthesis from cholesterol is upregulated in other endocrine cancers, as adrenocortical and ovarian carcinomas $[30,31]$. Intracellular cholesterol metabolism can also lead to the generation of a wide spectrum of oxysterols through the action of the cytochrome P450 family enzymes (CYPs). Interestingly, compared to parental non-tumor cells, PCa and BC cells could present with an altered expression of CYPs, leading to a different pattern of cholesterol metabolites; consequently, some cholesterol metabolites are overproduced in cancer cells, while other are generated in lower amounts [32]. Oxysterols are endogenous ligands of LXRs and are generally believed to exert anti-proliferative actions; LXR activation, besides regulating cholesterol homeostasis, triggers anti-inflammatory pathways in cancer and stromal cells [10,33]. For example, LXR agonists were shown to induce the release of anti-inflammatory interferon $\gamma$ from 
macrophages and T cells [34]. In addition, LXRs can increase the expression of sulfotransferases, leading to steroid inactivation [35]. The activity of oxysterols on cancer cells extends beyond LXR activation. Indeed, 7-ketocholesterol (as unsaturated fatty acids) was shown to act as ligand of the so-called antiestrogen binding site (AEBS); 5,6 $\alpha$ - and 5,6קepoxycholesterol and their condensation product with histamine, dendrogenin $A$, also displayed high AEBS affinity [36]. On the contrary, some oxysterols could contribute to tumor growth, as 27-hydroxycholesterol. Indeed, it was shown to specifically increase the growth of ER-positive BC cancer cells by acting as an ER agonist [32]. Interestingly, 27hydroxycholesterol accumulation has been described in ER-positive BC due to the downregulation of CYP7B1, blunting the transcription of many LXR-regulated genes and in particular of $A B C A 1$ [32]. Contrasting results were obtained for 27-hydroxycholesterol on $\mathrm{PCa}$ cells $[37,38]$.

Fatty acid metabolism by cancer and immune cells in the TME can also lead to the generation of bioactive molecules, such as eicosanoids [39]. These molecules are produced from arachidonic acid and other polyunsaturated fatty acids by the action of lipoxygenases and cyclooxygenases. Their role in cancer is still in debate since they were variably associated with cancer cell proliferation; indeed, while some eicosanoids may act as tumor suppressors (as omega-3 fatty acids and resolvins), others were shown to promote the survival and the proliferation of cancer cells [39]. In particular, prostaglandin E2 levels are elevated in several human malignancies, including BC, PCa and ovarian cancer, and were associated with poor prognosis and resistance to chemotherapy and radiotherapy [40,41]. Thromboxane A2 (TXA2) is also increasingly implicated in cancer progression, especially in triple negative BC. The binding of TXA2 to its receptor (thromboxane receptor, TP) enhances BC cell migration and invasion by triggering Rho activation [29]. Through the same mechanism, TP activation induced cytoskeletal reorganization of PCa cells [42]. On the contrary, resolvins may help to lower the risk of developing cancer [43]. These bioactive lipids, derived from the omega-3 fatty acids eicosapentaenoic acid and docosahexaenoic acid, are key players in the resolution of inflammation. In multiple tumor types, resolvins were shown to reduce tumor growth, neoangiogenesis, metastatization and to revert the deactivation of natural killer (NK) cells [44]. 
160 Along the mevalonate pathway, isoprenoids (farnesyl-pyrophosphate and geranylgeranyl-

161 pyrophosphate) are produced, which are needed for prenylation, a post-translational modification of proteins. Prenylation provides the target protein with a hydrophobic $\mathrm{C}$ terminus, which guides its localization within the cell, for example to the lipid rafts, favoring protein-protein interactions [45]. Many prenylated proteins are involved in cell signaling, as small GTP binding proteins. Among these, the oncogene RAS is the most studied and a reduction of isoprenoid production, as by farnesyltransferase inhibitors, is under investigation with promising results in aggressive BC [46].

Fatty acids are also involved in protein modification, as some proteins undergo myristoylation or palmitoylation $[47,48]$. Again, many of the target proteins are crucial components of signaling pathways and the modification with fatty acids promotes their binding to the membrane for proper localization and function. Well-characterized targets include many oncogene products, such as the tyrosine kinase Src $[47,48]$.

\section{Lipoproteins as pharmacological targets?}

\section{Epidemiologic evidence}

Several retrospective studies investigated the relationship between plasma levels of lipids/lipoproteins and the incidence of different cancer types. To date, conflicting results were obtained, since positive, negative or no relationships were found $[49,50]$. In the REduction by DUtasteride of prostate Cancer Events (REDUCE) trial, total cholesterol was positively associated with an increased risk of high-grade PCa (5\% higher for $10 \mathrm{mg} / \mathrm{dl})$, but not with low-grade one. HDL-C levels were also positively associated with overall risk of $\mathrm{PCa}(8 \%$ higher for $10 \mathrm{mg} / \mathrm{dl}$ ) [51]. However, in the same trial, men with high HDL-C displayed reduced prostate inflammation [52]. On the contrary, in the Alpha-Tocopherol, Beta-Carotene Cancer Prevention (ATBC) study, while the positive association between total cholesterol and the risk of advanced PCa was confirmed, HDL-C was negatively associated with PCa risk [53]. The association between plasma lipids and the risk of cancer was also investigated in prospective studies. Large meta-analyses excluded the association between plasma lipids and the risk of PCa and ovarian cancer, but HDL-C was negatively associated with BC risk [54-56]. plasma lipids and $\mathrm{PCa}$ or $\mathrm{BC}$ risk. No association of plasma lipids with $\mathrm{PCa}$ risk was found, 
192 [57]. On the contrary, genetically raised LDL-C levels were associated with higher risk of BC, 193 especially of the ER-positive type [58]. The same relationship was found for elevated HDL-C, 194 but it should be noted that only the effect of CETP variants was assessed, and inhibiting CETP is debated for the possible accumulation of dysfunctional HDL [59]. The role of dietary lipids on cancer risk is also debated, since a lipid-rich diet could be indicative of an unhealthy lifestyle. In addition, a significant association between dietary cholesterol intake and the risk of $B C$ was detected only with very high intakes [60]. Another approach to address the relationship between plasma lipids and the incidence of cancer is to assess the association with statins. In two recent meta-analysis, statins were shown to have a neutral effect on both PCa and BC incidence $[61,62]$. Consistently, the Cholesterol Treatment Trialists' Collaboration showed no evidence of any effect of reducing cholesterol with long-term statin therapy on cancer incidence or mortality [63]. To explain the inconsistency between studies, the type of cancer and the time at which lipids were assessed could act as confounding factors. The stage of the disease and the consequent therapies can affect the levels of circulating lipids; plasma lipids, especially HDL-C levels, are reduced in several cancer patients during active disease, as in those having breast, ovarian, prostate, colon and pancreatic carcinomas [64]. The large HDL2 subclass is particularly reduced [65]. The predictive power of low HDL-C and apoA-I levels was so strong that apoA-I was included in the screening for ovarian cancer with other traditional biomarkers [66]. The mechanisms beyond HDL-C reduction are not fully understood. Since the liver and the intestine are responsible for the synthesis of apoA-I and its lipidation by ABCA1, malignancies at these organs can directly impaired HDL biogenesis. As shown in many inflammatory states, proinflammatory cytokines inhibit hepatic apoA-I expression and, thus, the cancer-related sustained inflammation can repress HDL biogenesis by the same mechanism [67]. Finally, the hepatic expression of several proteins involved in lipoprotein metabolism can be modulated by inflammation and cancer, including LCAT; thus, HDL-C reduction could be part of a more complex alteration of lipoprotein metabolism induced by cancer [67]. The association between plasma lipids and the recurrence of cancer has been firmly established. Plasma levels of cholesterol are positively associated with a higher recurrence rate of $B C$ and statin use was associated with extended recurrence free survival time [68]. This protective effect was confirmed in women with hormone receptor-positive early-stage breast cancer taking statins during adjuvant endocrine therapy [69]. Plasma levels of HDL-C 
224 are inversely related to the prognosis; in a recent meta-analysis including both retrospective 225 and prospective studies, patients with higher HDL-C had $37 \%$ reduced risk of death and $35 \%$ 226 reduced risk of disease relapse compared to patients with lower HDL-C [70].

\section{Targeting apoB-containing lipoproteins}

The use of hyperlipidemic mice (apoE $/$ model) fed a high fat/high cholesterol diet (HFHC) can help to address the impact of increased plasma levels of apoB-containing lipoproteins on the development of hormone-dependent cancers. The injection of non-metastatic Met-1 and metastatic Mvt-1 mammary cancer cells in apoE $E^{-/}$mice resulted in larger tumors and in a greater number of lung metastases compared to wild-type mice [71]. Interestingly, the graft with BC cell lines representative of triple-negative or HER2-enriched tumors in hyperlipidemic mice resulted in reduced tumor growth when the LDL-R was silenced [72]. Consistently, LDL increased the proliferation and migration of ER-negative BC cells but not of ER-positive ones, which also accumulated less CEs than ER-negative cells [73]. These data suggest that ERnegative BC may be more sensitive to cell cholesterol-lowering strategies. Regarding PCa, mice fed a HFHC diet developed larger tumors after injection of androgen-dependent LNCaP cells compared to mice under chow diet [24]. In addition, the HFHC diet accelerated tumor incidence and burden compared to chow diet in the TRAMP mouse, a model of PCa [74]. These data are supported by several in vitro studies. VLDL, but not LDL, increased the formation of BC cell mammospheres, an estimate of stem cell/early progenitor activity, and cell resistance to radiotherapy, likely due to a modulation of intracellular cholesterol content [75]. Consistently, the treatment of aggressive and metastatic BC cells with statins was associated with an increased sensitivity to radiation therapy [75]. The in vivo relevance of these findings was supported by the shorter recurrence-free period in patients with elevated VLDL-C levels, which suggests a systemic effect of statins through the reduction of circulating apoB-containing lipoproteins [75]. Oxidized LDL (oxLDL), but not native LDL, increased the proliferation of ovarian cancer cells and decreased their sensitivity to cisplatin, an effect prevented by statins and by LXR activation [76]. OxLDL could act through the binding with its lectin-like oxidized low-density lipoprotein receptor-1 (LOX-1), which stimulates the expression of pro-adhesive, pro-inflammatory and proangiogenic factors in vascular endothelial cells and macrophages. LOX-1 upregulation was reported in several different types of cancer, including PCa. Here, its activation by oxLDL promoted epithelial to 
mesenchymal transition and increased tumor growth in nude mice [77]. In BC cell lines, LOX-

2571 inhibition blunted inflammatory and hypoxic responses [78]. Finally, apoB-containing 258 lipoproteins and adipocytes could deliver free fatty acids to cancer cells by the action of 259 locally expressed LPL; BCs are generally positive for LPL and its expression increased cell 260 proliferation [79]. Moreover, adipocytes can directly provide cancer cells with fatty acids. 261 Indeed, cancer cells can stimulate TG lipolysis by the adipose triglyceride lipase/hormone262 263 264 265 266 267 268

\section{Targeting the HDL system}

Animal studies provided proofs of a causal link between HDL and cancer risk. When HDL-C levels were increased by genetic manipulation (as in the human apoA-I transgenic mice) or by direct infusion of apoA-I, the growth of lung, melanoma or ovarian cancer cells after xenograft was reduced compared to control animals [88]. Consistently, in apoA-1/- mice, in which HDL-C levels are dramatically reduced, tumor development increased compared to wild-type animals 
287 [88]. More interestingly, apoA-I infusion after the development of the tumor mass resulted in 288 tumor shrinkage [88].

289 In vitro studies provided some mechanistic insights. When BC cells were incubated with HDL, 290 the formation of mammospheres was inhibited and their sensitivity to radiation therapy 291 increased, an effect due to a reduced cholesterol content [75]. Whether this reduction was 292 ascribed to a depletion of cell membrane content or of intracellular CE stores in LDs was not 293 addressed. It could be speculated that, given the continual cycle of hydrolysis and esterification of CE in LDs, the removal of unesterified cholesterol from the cell membrane 295 promoted by HDL could shift the balance towards CE hydrolysis with a consequent LD 296 depletion, as shown in macrophages [89]. In PCa cell lines, $\mathrm{HDL}$ blunted basal and $\mathrm{H}_{2} \mathrm{O}_{2}-$ induced oxidative stress and reduced ROS-induced proliferation, with a role for both the 298 protein and phospholipid components [90].

299 On the contrary, some studies showed a SR-BI-dependent increased growth of cancer cells 300 incubated with HDL [91,92]. In this context, large CE-enriched HDL could represent a better 301 substrate for SR-BI-mediated cholesterol influx supporting cancer cell growth. It has to be 302 pointed out that HDL could become dysfunctional in several pathologic conditions, including inflammatory states, metabolic diseases and cancer, due to modifications of their protein and lipid cargo [64,93]. Consistently, the proliferation of the BC cell line MCF-7 was induced by $\mathrm{HDL}$ isolated from type 2 diabetic patients, but not by HDL from healthy controls. Only HDL from diabetic patients were able to promote $\mathrm{BC}$ metastasis by increasing the adhesion of cancer cells to endothelial cells $[94,95]$.

Two approaches can be used to raise circulating HDL. The first is to improve HDL biogenesis or to limit their catabolism. To date, no drug specifically increases HDL-C levels. Fibrates and niacin have multiple effects on lipid metabolism including the increase of HDL biogenesis, but their role in cancer management has not been investigated. CETP inhibitors specifically increase HDL-C, but their development is hampered by the lack of a clear cardiovascular benefit, together with the possibility to accumulate dysfunctional HDL [59]. The second approach is to infuse reconstituted HDL (rHDL), discoidal particles made of phospholipids and apoA-I (or synthetic apoA-I mimetic peptides) that were shown to retain the atheroprotective activities of HDL [96]. Reconstituted HDL have been already tested in multiple in vivo and in vitro tumor models [97]. Among the underlying mechanisms, anti-inflammatory activities, inhibition of angiogenesis, abrogation of growth factor-induced proliferation, migration and 
invasion were described [97]. Multiple positive effects of rHDL on TME cellular composition were also shown, as a reduced content of myeloid-derived suppressor cells and the accumulation of M1 macrophages and cytotoxic CD8+ T cells [88]. Some of these effects were detected with direct apoA-I or apoA-I mimetic peptide administration, suggesting an antitumor activity of HDL apolipoprotein components [88]; however, it cannot be fully excluded that apoA-I or its peptides could rapidly acquire phospholipids and cholesterol in the circulation, thus generating HDL-like particles.

HDL-like particles for drug delivery

Reconstituted HDL are also under development as drug carriers for tumor targeting. They accumulate within the tumor mass by unselective mechanisms (as nanoparticles do through the leaky vasculature) and by the specific interaction with SR-BI. This receptor is highly expressed in multiple cancers and allows cytosolic delivery of the payload bypassing the endolysosomal route [98]. Unlike exogenous nanoparticles, rHDL containing apoA-I are nonimmunogenic and have long circulation half-life. In addition, the small diameter $(<14 \mathrm{~nm})$ allows a deeper penetration of rHDL into the tumor mass if compared to nanoparticles, which is crucial for the treatment of solid tumors [99].

Several types of molecules can be incorporated into rHDL as small molecule drugs, small interfering RNAs, photothermal agents or fluorescent dyes for imaging [98]. The composition and the particle size/shape of rHDL can be tailored according to the type of molecule to be carried and the tumor to be targeted. For example, spherical rHDL were used for the encapsulation of highly hydrophobic drugs into the particle core: by this way paclitaxel and valrubicin were successfully delivered to $\mathrm{PCa}$, triple-negative $\mathrm{BC}$ or ovarian cancer cells in 342 vitro and in vivo [100-102].

\section{Concluding remarks and future perspectives}

345 Targeting the lipoprotein system is becoming an attractive approach to develop novel 346 therapeutic strategies for the management of hormone-dependent cancers. The conflicting results of epidemiological and interventional studies suggest that the availability of lipoproteins in the TME is likely more relevant than their circulating levels. In this context, statins use seems not a promising strategy due to their preferential hepatic distribution and upregulation of the LDL-R expression. On the contrary, targeted repression of the LDL-R in 
351 cancer cells, as by genetic silencing, already generated positive results in different types of 352 cancer. Another approach is to increase the efficiency of the HDL system in promoting lipid 353 removal, as by the infusion of rHDL to rise the endogenous HDL pool. This approach has 354 some advantages. Reconstituted HDL are already under clinical development for patients with 355 acute coronary syndrome [96]. Their protein and lipid composition can be manipulated to 356 optimize their function. In addition, rHDL can be used as carriers of other bioactive molecules, 357 ranging from nucleic acids to drugs [98].

358 Overall, targeting lipid metabolism in the TME should be considered as an adjuvant strategy 359 to increase cancer cell sensitivity to classical therapeutic agents. 
360 Figure legends

361 Figure 1. Cholesterol metabolism and function in cancer cells.

362 Cholesterol can be synthetized from acetyl-CoA through the mevalonate pathway or it can be 363 taken up from lipoproteins in the tumor microenvironment. Indeed, very low density 364 lipoproteins (VLDL), low density lipoproteins (LDL) and their remnants can undergo 365 endocytosis mediated by the LDL-receptor family. On the contrary, high density lipoproteins 366 (HDL) promote cholesterol efflux from cancer cells through their interaction with ATP-binding 367 cassette transporters $A 1$ and $\mathrm{G} 1$ (ABCA1, ABCG1) or with the scavenger receptor type $\mathrm{BI}$ 368 (SR-BI). Since SR-BI mediates a facilitated diffusion of cholesterol according to concentration 369 gradient, it could also favor lipid influx. Cholesterol is a key component of lipid rafts, and it can 370 be converted to hormones and oxysterols. Created with BioRender.

372 Figure 2. Lipid homeostasis in cancer cells.

373 Cellular homeostasis of lipids is regulated by sterol regulatory element-binding proteins 374 (SREBPs) and liver X receptors (LXRs), according to the metabolic needs of the cell. In 375 cancer cells, the balance between SREBPs and LXRs is lost, with an hyperactivation of 376 SREBPs and LXR inhibition. Consequently, cholesterol and fatty acid synthesis is 377 upregulated, as the uptake of lipoproteins; on the contrary, cholesterol efflux is blunted. 378 Created with BioRender. 


\section{Box 1. Incidence and classification of breast and prostate cancers}

380 Breast cancer is the most common cancer in women and the second most common cancer overall. Age-standardized incidence rate in Europe, North America and Australia is $>70$ cases per 100.000 women [103]. BC is highly heterogeneous and it is currently classified according to histopathological features and gene expression profiling into luminal A, luminal B, HER2enriched and basal-like (commonly referred to as triple-negative) (Table 1) [104]. This classification could predict the clinical outcome and the response to therapeutic interventions. Luminal BCs, which are positive to estrogen and/or progesterone receptors, will likely respond to hormone therapy; HER2-targeted treatments are indicated in HER2-enriched BC, while conventional chemotherapy is used for basal-like $\mathrm{BC}$ [1].

Table 1. Classification of BC

\begin{tabular}{|l|c|c|c|}
\hline & ER/PR & HER2 & Ki67 \\
\hline Luminal A & + & - & $<14 \%$ \\
\hline Luminal B & + & - & $>14 \%$ \\
\hline HER2-enriched & - & + & \\
\hline Basal-like & - & - & \\
\hline
\end{tabular}

ER, estrogen receptor; PR, progesterone receptor; HER2, epidermal growth factor type II receptor; Ki67, nuclear protein used as a proliferation marker.

Prostate cancer is the most common cancer in men and the fourth most common cancer overall. Age-standardized incidence rate in Europe, North America and Australia is $>60$ cases per 100.000 men [103]. The morphological classification of PCa has been significantly modified in the last decade [105]. The new grading system into 5 categories is based on the relative prevalence of (i) well-formed glands, (ii) poorly formed, fused or cribriform glands, (iii) lack of gland formation or necrosis. This classification is integrated with prostate-specific antigen levels and with the following information: (i) localization and size of the tumor mass, (ii) whether the cancer has spread to nearby lymph nodes, (iii) whether the cancer has metastasized. The final result is PCa staging from I to IV, which guides the selection of treatment approach among active surveillance, prostatectomy, radiotherapy, hormone- 
406 Normal cells rely on fatty acids oxidation (FAO) and mitochondrial oxidative phosphorylation 407 (OXPHOS) for acetyl-CoA and ATP production. Cancer cells rewire their energetic machinery 408 from OXPHOS to anaerobic glycolysis, especially in the hypoxic core of solid tumors; 409 glycolysis occurs even in the presence of oxygen (the Warburg effect), generating large 410 amounts of lactate that contributes to TME acidity [106]. Altered tumor cell metabolism affects 411 TME cells; in particular, the release of lactate and TME acidification inhibit dendritic and T cell 412 activation, thus favoring tumor immune escape [107]. Interestingly, ATP is still produced in the 413 mitochondria of tumor cells and mitochondrial tricarboxylic acid cycle intermediates are 414 needed as precursors for macromolecule synthesis, as citrate for lipid synthesis and 415 oxaloacetate for nucleotide synthesis [106]. In this context, FAO is the main source of acetyl416 CoA for mitochondrial OXPHOS.

417 Interestingly, low rates of glycolysis were found in PCa and, indeed, PCa cells were shown to 418 have a dominant uptake of fatty acids over glucose [108]. In addition, stromal cells and, most 419 interestingly, cancer stem cells still rely on OXPHOS for energy production. The latter are now widely considered having a strong metastatic potential and resistance to radiotherapy 421 and chemotherapy [109].

\section{Box 3. Structure and metabolism of lipoproteins}

Lipoproteins carry lipids in the circulation; they are composed by a core of hydrophobic triglycerides (TG) and cholesteryl esters (CE), surrounded by a double layer of phospholipids, unesterified cholesterol and apolipoproteins. According to their density and apolipoprotein composition, they are classified into chylomicrons, very low density lipoproteins (VLDL), low density lipoproteins (LDL) and high density lipoproteins (HDL), which have distinct roles in lipid metabolism [110].

Chylomicrons are secreted by intestinal epithelial cells and transport dietary lipids to the liver. They are the largest and less dense lipoproteins, because their lipid cargo is mainly composed by TG. Their main protein component is apoB-48. After TG hydrolysis by lipoprotein lipase (LPL) and the release of free fatty acids to peripheral tissues, the liver takes up chylomicron remnants through the interaction of apoE with members of the LDL receptor family (LDL-R and the LDL-related receptor protein, LRP-1). 
VLDL are secreted by the liver and are enriched in TG; their main protein component is apoB100. After TG hydrolysis by LPL, generated remnants are converted into LDL, whose core is mainly composed by CE. LDL deliver cholesterol to peripheral tissues by the interaction of apoB-100 with LDL-R. Circulating LDL can be taken up by the liver through the same mechanism. The liver also secretes lipoprotein (a) (Lp(a)), which is a LDL-like particle with one molecule of apo(a) covalently bound to apoB. Apo(a) is structurally similar to plasminogen, with multiple copies of kringle domains, but lacks the fibrinolytic activity; thus, by competing with plasminogen, apo(a) could exert a potential pro-thrombotic effect [111]. 444 The biogenesis of HDL is more complex [112]. Their main protein component, apoA-I, is secreted by the liver and by the intestine and is rapidly lipidated by the interaction with the $A B C$ transporter $A 1$ ( $A B C A 1$ ) and the formation of discoidal nascent HDL. Further uptake of cholesterol and phospholipids and cholesterol esterification by lecithin:cholesterol acyltransferase (LCAT) leads to the formation of mature spherical HDL. In addition, HDL can be generated from the dissociation of lipids and apoA-I during the remodeling of apoBcontaining lipoproteins. The main role of HDL is to deliver cholesterol to the liver. The first step of this process is the efflux of cholesterol and phospholipids from cell membranes to HDL or apoA-I [13]. Cholesterol efflux can (i) occur by passive diffusion, (ii) be actively promoted by $A B C A 1$ and $A B C G 1$, or (iii) occur by diffusion facilitated by the scavenger receptor type $\mathrm{BI}$ (SR-BI). Thus, according to the gradient concentration between cell membranes and HDL, SR-BI can mediate cholesterol efflux or influx. Once cholesterol is esterified by LCAT, HDL can directly deliver CE to the liver through hepatic SR-BI, which mediates the selective uptake of $\mathrm{CE}$ without $\mathrm{HDL}$ endocytosis. However, in humans the majority of $\mathrm{CE}$ is indirectly routed to the liver by the action of CE transfer protein (CETP), which mediates the exchange of CE for TG between HDL and apoB-containing lipoproteins. CE are then taken up by the liver through the endocytosis of apoB-containing lipoproteins by LDL-R and LRP-1.

\section{Box 4. Effects of lipoproteins on cell metabolism: insights from cardiovascular} diseases

Most of the available evidence on the role of lipoproteins in cell metabolism comes from the field of vascular biology and atherosclerosis. Lipoproteins can be divided into proatherogenic (apoB-containing lipoproteins, i.e. VLDL, LDL, Lp(a) and TG-rich remnants) and 
468 atherosclerosis because they mediate the transport of lipids from the liver to peripheral 469 tissues, including the arterial wall [113]. On the contrary, HDL are the main acceptors of 470 cholesterol from peripheral tissues and mediate its transport to the liver for excretion, among 471 the so-called "reverse cholesterol transport" [13]. While systemic levels of lipids can widely 472 vary in different physiologic and pathologic conditions, their cell content is tightly regulated 473 [114,115]. Cells can synthesize cholesterol and fatty acids or lipids can be taken up from 474 circulating lipoproteins. However, excess cholesterol is toxic for the cells and, consequently, it 475 is removed through efflux towards extracellular acceptors as HDL, or it is esterified by acyl476 coA:cholesterol acyltransferase (ACAT) and stored in cytosolic lipid droplets (LDs); the same 477 happens to fatty acids, which are stored in LDs as TG. The cellular sensors of cholesterol and 478 fatty acid content, that regulate the pathways described above, are the sterol regulatory 479 element binding proteins (SREBPs) and the liver $\mathbf{X}$ receptors (LXRs) $[114,115]$. When 480 cells need cholesterol and/or fatty acids, SREPBs are activated, promoting lipid synthesis and 481 uptake, while LXRs are inhibited. On the contrary, when intracellular levels of lipids are 482 increased, SREBPs are switched off and LXRs activated. LXRs favor lipid efflux by increasing 483 the expression of the $A B C$ transporters, and they decrease lipoprotein uptake through the 484 downregulation of the LDL-R (see box 3) [116,117]. However, scavenger receptors, as the 485 cluster of differentiation 36 (CD36), can mediate an unregulated uptake of lipoproteins and, 486 consequently, an excessive lipid accumulation in macrophages within the arterial wall [118]. 487 The role of lipoproteins is complex and not limited to the in/out transport of lipids. ApoB488 containing lipoproteins, including TG-rich remnants, exert pro-inflammatory activities on 489 different cell types, especially after oxidation [17]. On the contrary, HDL were shown to exert 490 a series of anti-inflammatory and antioxidant activities on several cells involved in the 491 atherosclerotic process [119]. In addition, lipoproteins, especially HDL, can act as carriers of 492 several bioactive molecules, including miRNAs [120]. 


\section{Glossary}

494 Antiestrogen binding site (AEBS): It has been identified as a microsomal high-affinity

495 binding site for the estrogen antagonist tamoxifen, distinct from the estrogen receptor. It does 496 not bind estrogens. AEBS is composed by the $3 \beta$-hydroxysterol- $\Delta^{8}-\Delta^{7}$-isomerase (D8D7I) and 497 the 7-dehydrocholesterol reductase (DHCR7) enzymes, which are involved in cholesterol 498 synthesis.

499 Atherosclerosis: Pathologic process characterized by the accumulation of lipids and cells in 500 the arterial wall, causing a progressive narrowing of the arterial lumen.

501 Cancer stem cells: Cancer cells expressing markers of hematopoietic stem cells, which are 502 able to initiate tumors in vivo. Their presence was shown in all solid tumors.

503 Cytochrome P450 enzymes (CYPs): A family of oxidative enzymes involved in the synthesis 504 and metabolism of various molecules within the cells. They are essential for the metabolism 505 and excretion of xenobiotics. The liver is enriched in CYPs, but they are expressed 506 throughout the body.

507 Glycolysis: A sequence of reactions that converts glucose into pyruvate.

508 Hypoxia: A condition in which local oxygen supply is insufficient for cell metabolic 509 requirements. The TME becomes hypoxic due to the fast proliferation of cancer cells that is 510 not supported by an adequate formation of novel vessels. When the tumor mass grows, 511 oxygen delivered by blood is quickly consumed by cancer cells that are closest to the vessels, 512 thus hampering its diffusion into the tumor mass. Consequently, most solid tumors display 513 regions that are permanently or transiently in hypoxic conditions.

514 Lipid droplets: Intracellular stores of fatty acids and cholesterol in the form of neutral lipids.

515 They are hydrolyzed in the cytosol or in the lysosomes to meet cell energy requirements.

516 Liver $X$ receptors (LXRs): Nuclear receptors activated by oxysterols. $L X R \alpha$ is mainly 517 expressed in the liver, while LXR $\beta$ is ubiquitously expressed. LXRs play a crucial role in cell 518 metabolism since they control the transcription of genes involved in cholesterol, fatty acid and 519 glucose homeostasis.

520 Mendelian randomization studies: Epidemiologic studies in which genetic variations are 521 used to investigate the causal link between a potentially modifiable risk factor and disease 522 outcome. 
523 Oxysterols: Oxygenated derivatives of cholesterol produced by enzymatic or nonenzymatic 524 peroxidation. They are intermediates or end-products of cholesterol excretion by its 525 transformation into water-soluble bile acids. Oxysterols regulate cholesterol homeostasis and 526 can exert potent effects on several biological processes.

527 RAS: Oncogene coding for small GTP binding proteins involved in the regulation of the cell 528 proliferation and death. Members of the RAS family include KRAS, HRAS, and NRAS. RAS is 529 frequently mutated in human cancers.

530 Statins: Lipid-lowering agents that inhibit the 3-hydroxy-3-methyglutaryl coenzyme A (HMG$531 \mathrm{CoA}$ ) reductase, the rate-limiting enzyme of cholesterol synthesis along the mevalonate 532 pathway.

533 Sterol regulatory element-binding proteins (SREBPs): Family of transcription factors 534 consisting of two genes, SREBF1 and SREBF2, that encode for three different proteins: 535 SREBP1a, SREBP1c and SREBP2. They regulate the transcription of genes involved in 536 cholesterol biosynthesis and uptake, and fatty acid biosynthesis. 
References

1. Senkus, E. et al. (2015) Primary breast cancer: ESMO Clinical Practice Guidelines for 540 diagnosis, treatment and follow-up. Ann. Oncol. 26 Suppl 5, v8-30

2. Cornford, P. et al. (2017) EAU-ESTRO-SIOG Guidelines on Prostate Cancer. Part II:

Treatment of Relapsing, Metastatic, and Castration-Resistant Prostate Cancer. Eur. Urol. 71, 630-642

3. Quail, D.F. and Joyce, J.A. (2013) Microenvironmental regulation of tumor progression and metastasis. Nat. Med. 19, 1423-1437

546

4. Hanahan, D. and Coussens, L.M. (2012) Accessories to the crime: functions of cells recruited to the tumor microenvironment. Cancer Cell 21, 309-322

5. Mittal, S. et al. (2018) The breast tumor microenvironment: role in cancer development, 549 progression and response to therapy. Expert. Rev. Mol. Diagn. 18, 227-243

550 6. Finak, G. et al. (2008) Stromal gene expression predicts clinical outcome in breast cancer. $551 \quad$ Nat. Med. 14, 518-527

552

7. Farmer, P. et al. (2009) A stroma-related gene signature predicts resistance to neoadjuvant 553 chemotherapy in breast cancer. Nat. Med. 15, 68-74

554 8. Grivennikov, S.I. et al. (2010) Immunity, inflammation, and cancer. Cell 140, 883-899

555 9. Pike, L.J. (2003) Lipid rafts: bringing order to chaos. J. Lipid Res. 44, 655-667

556 10. Schroepfer, G.J., Jr. (2000) Oxysterols: modulators of cholesterol metabolism and other 557 processes. Physiol Rev. 80, 361-554

558 11. Krycer, J.R. and Brown, A.J. (2013) Cholesterol accumulation in prostate cancer: a classic 559 observation from a modern perspective. Biochim. Biophys. Acta 1835, 219-229

560 12. Menard, J.A. et al. (2018) Functional role of extracellular vesicles and lipoproteins in the 561

562 13. Rosenson, R.S. et al. (2012) Cholesterol efflux and atheroprotection: advancing the 563 concept of reverse cholesterol transport. Circulation 125, 1905-1919

14. Chen, Y. and Hughes-Fulford, M. (2001) Human prostate cancer cells lack feedback regulation of low-density lipoprotein receptor and its regulator, SREBP2. Int. J. Cancer 91, 4145

15. Chuu, C.P. et al. (2006) Inhibition of tumor growth and progression of LNCaP prostate cancer cells in athymic mice by androgen and liver X receptor agonist. Cancer Res. 66, 64826486 
16. Menard, J.A. et al. (2016) Metastasis Stimulation by Hypoxia and Acidosis-Induced

Extracellular Lipid Uptake Is Mediated by Proteoglycan-Dependent Endocytosis. Cancer Res. $76,4828-4840$

573 17. Pirillo, A. et al. (2013) LOX-1, OxLDL, and atherosclerosis. Mediators. Inflamm. 2013, $574 \quad 152786$

575

576

577

578

579

580

581

582

583

584

585

586

587

588

589

590

591

592

593

594

595

596

597

598

599

600

601

602

603

604

18. de Gonzalo-Calvo, D. et al. (2015) Intratumor cholesteryl ester accumulation is associated with human breast cancer proliferation and aggressive potential: a molecular and clinicopathological study. BMC. Cancer 15, 460

19. Pelton, K. et al. (2012) Cholesterol and prostate cancer. Curr. Opin. Pharmacol. 12, 751 759

20. Chen, M. et al. (2018) An aberrant SREBP-dependent lipogenic program promotes metastatic prostate cancer. Nat. Genet. 50, 206-218

21. Griffiths, B. et al. (2013) Sterol regulatory element binding protein-dependent regulation of lipid synthesis supports cell survival and tumor growth. Cancer Metab 1, 3

22. Yue, S. et al. (2014) Cholesteryl ester accumulation induced by PTEN loss and PI3K/AKT activation underlies human prostate cancer aggressiveness. Cell Metab 19, 393-406

23. John, S. et al. (2017) Extracellular Proton Concentrations Impacts LN229 Glioblastoma Tumor Cell Fate via Differential Modulation of Surface Lipids. Front Oncol. 7, 20

24. Zhuang, L. et al. (2005) Cholesterol targeting alters lipid raft composition and cell survival in prostate cancer cells and xenografts. J. Clin. Invest. 115, 959-968

25. Ushio-Fukai, M. et al. (2001) Cholesterol Depletion Inhibits Epidermal Growth Factor Receptor Transactivation by Angiotensin II in Vascular Smooth Muscle Cells. Role of cholesterol-rich microdomains and focal adhesions in angiotensin II signaling. J. Biol. Chem. 276, 48269-48275

26. Simons, K. and Ehehalt, R. (2002) Cholesterol, lipid rafts, and disease. J. Clin. Invest. $110,597-603$

27. Wang, X. et al. (2015) Aromatase overexpression in dysfunctional adipose tissue links obesity to postmenopausal breast cancer. J. Steroid Biochem. Mol. Biol. 153, 35-44

28. Mitsiades, N. et al. (2012) Distinct patterns of dysregulated expression of enzymes involved in androgen synthesis and metabolism in metastatic prostate cancer tumors. Cancer Res. 72, 6142-6152

29. Goss, P.E. et al. (2016) Extending Aromatase-Inhibitor Adjuvant Therapy to 10 Years. $N$. Engl. J. Med. 375, 209-219

30. Clinton, G.M. and Hua, W. (1997) Estrogen action in human ovarian cancer. Crit Rev. Oncol. Hematol. 25, 1-9 
31. Imachi, H. et al. (1999) Evidence for a potential role for HDL as an important source of cholesterol in human adrenocortical tumors via the CLA-1 pathway. Endocr. J. 46, 27-34

607 32. Nelson, E.R. et al. (2014) Cholesterol and breast cancer pathophysiology. Trends 608 Endocrinol. Metab 25, 649-655

609

610

611 612

613 614

615

616

617

618 619

620

621

622

623

624

625

626

627

628

629

630

631

632

633

634

635

636

637

33. York, A.G. and Bensinger, S.J. (2013) Subverting sterols: rerouting an oxysterol-signaling pathway to promote tumor growth. J. Exp. Med. 210, 1653-1656

34. Wang, Q. et al. (2014) Identification of interferon-gamma as a new molecular target of liver X receptor. Biochem. J. 459, 345-354

35. Lee, J.H. et al. (2008) Androgen deprivation by activating the liver $\mathrm{X}$ receptor.

Endocrinology 149, 3778-3788

36. Leignadier, J. et al. (2017) Improving the efficacy of hormone therapy in breast cancer: The role of cholesterol metabolism in SERM-mediated autophagy, cell differentiation and death. Biochem. Pharmacol. 144, 18-28

37. Alfaqih, M.A. et al. (2017) CYP27A1 Loss Dysregulates Cholesterol Homeostasis in Prostate Cancer. Cancer Res. 77, 1662-1673

38. Raza, S. et al. (2017) The cholesterol metabolite 27-hydroxycholesterol stimulates cell proliferation via ERbeta in prostate cancer cells. Cancer Cell Int. 17, 52

39. Wang, D. and Dubois, R.N. (2010) Eicosanoids and cancer. Nat. Rev. Cancer 10, 181193

40. Tong, D. et al. (2018) The roles of the COX2/PGE2/EP axis in therapeutic resistance. Cancer Metastasis Rev. 37, 355-368

41. Reinartz, S. et al. (2016) A transcriptome-based global map of signaling pathways in the ovarian cancer microenvironment associated with clinical outcome. Genome Biol. 17, 108

42. Nie, D. et al. (2008) Thromboxane A2 receptors in prostate carcinoma: expression and its role in regulating cell motility via small GTPase Rho. Cancer Res. 68, 115-121

43. Zhang, Q. et al. (2017) Resolution of Cancer-Promoting Inflammation: A New Approach for Anticancer Therapy. Front Immunol. 8, 71

44. Sulciner, M.L. et al. (2018) Targeting lipid mediators in cancer biology. Cancer Metastasis Rev. 37, 557-572

45. Wang, M. and Casey,P.J. (2016) Protein prenylation: unique fats make their mark on biology. Nat. Rev. Mol. Cell Biol. 17, 110-122

46. Karp, J.E. and Lancet, J.E. (2007) Development of farnesyltransferase inhibitors for clinical cancer therapy: focus on hematologic malignancies. Cancer Invest 25, 484-494 
48. Lanyon-Hogg, T. et al. (2017) Dynamic Protein Acylation: New Substrates, Mechanisms, and Drug Targets. Trends Biochem. Sci. 42, 566-581

49. Danilo, C. and Frank, P.G. (2012) Cholesterol and breast cancer development. Curr.

50. De Boussac, H. et al. (2013) LXR, prostate cancer and cholesterol: the Good, the Bad

51. Jamnagerwalla, J. et al. (2018) Serum cholesterol and risk of high-grade prostate cancer: results from the REDUCE study. Prostate Cancer Prostatic. Dis. 21, 252-259

647 52. Allott, E.H. et al. (2017) Statin Use, Serum Lipids, and Prostate Inflammation in Men with a Negative Prostate Biopsy: Results from the REDUCE Trial. Cancer Prev. Res. (Phila) 10, 319-326

53. Mondul, A.M. et al. (2011) Serum total and HDL cholesterol and risk of prostate cancer.

\section{Cancer Causes Control 22, 1545-1552}

54. YuPeng, L. et al. (2015) Cholesterol Levels in Blood and the Risk of Prostate Cancer: A Meta-analysis of 14 Prospective Studies. Cancer Epidemiol. Biomarkers Prev. 24, 1086-1093

55. Touvier, M. et al. (2015) Cholesterol and breast cancer risk: a systematic review and Review and Dose-Response Meta-Analysis of Observational Studies. Nutr. Cancer 71, 939953

57. Bull, C.J. et al. (2016) Blood lipids and prostate cancer: a Mendelian randomization analysis. Cancer Med. 5, 1125-1136

58. Nowak, C. and Arnlov, J. (2018) A Mendelian randomization study of the effects of blood lipids on breast cancer risk. Nat. Commun. 9, 3957

59. Yamashita, S. and Matsuzawa, Y. (2016) Re-evaluation of cholesteryl ester transfer protein function in atherosclerosis based upon genetics and pharmacological manipulation.

Curr. Opin. Lipidol. 27, 459-472

60. Li, C. et al. (2016) Systematic review and meta-analysis suggest that dietary cholesterol intake increases risk of breast cancer. Nutr. Res. 36, 627-635 definitive therapy: a systematic review and meta-analysis. Sci. Rep. 6, 29106

670 62. Undela, K. et al. (2012) Statin use and risk of breast cancer: a meta-analysis of 671 observational studies. Breast Cancer Res. Treat. 135, 261-269 
63. Emberson, J.R. et al. (2012) Lack of effect of lowering LDL cholesterol on cancer: metaanalysis of individual data from 175,000 people in 27 randomised trials of statin therapy.

674 PLOS. ONE. 7, e29849

675 64. Ganjali, S. et al. (2019) High-Density Lipoprotein Components and Functionality in 676 Cancer: State-of-the-Art. Trends Endocrinol. Metab 30, 12-24

677

678

679

680

681

682

683

684

685

686

687

688

689

690

691

692

693

694

695

696

697

698

699

700

701

702

703

704

705

706

707

65. Michalaki, V. et al. (2005) Evaluation of serum lipids and high-density lipoprotein subfractions (HDL2, HDL3) in postmenopausal patients with breast cancer. Mol. Cell Biochem. 268, 19-24

66. Kozak, K.R. et al. (2003) Identification of biomarkers for ovarian cancer using strong anion-exchange ProteinChips: potential use in diagnosis and prognosis. Proc. Natl. Acad. Sci. U. S. A. $100,12343-12348$

67. Pirro, M. et al. (2018) High density lipoprotein cholesterol and cancer: Marker or causative? Prog. Lipid Res. 71, 54-69

68. Mansourian, M. et al. (2016) Statins Use and Risk of Breast Cancer Recurrence and Death: A Systematic Review and Meta-Analysis of Observational Studies. J. Pharm. Pharm. Sci. $19,72-81$

69. Borgquist, S. et al. (2017) Cholesterol, Cholesterol-Lowering Medication Use, and Breast Cancer Outcome in the BIG 1-98 Study. J. Clin. Oncol. 35, 1179-1188

70. Zhou, P. et al. (2018) Prognostic role of serum total cholesterol and high-density lipoprotein cholesterol in cancer survivors: A systematic review and meta-analysis. Clin. Chim. Acta 477, 94-104

71. Alikhani, N. et al. (2013) Mammary tumor growth and pulmonary metastasis are enhanced in a hyperlipidemic mouse model. Oncogene 32, 961-967

72. Gallagher, E.J. et al. (2017) Elevated tumor LDLR expression accelerates LDL cholesterol-mediated breast cancer growth in mouse models of hyperlipidemia. Oncogene 36, 6462-6471

73. Antalis, C.J. et al. (2010) High ACAT1 expression in estrogen receptor negative basal-like breast cancer cells is associated with LDL-induced proliferation. Breast Cancer Res. Treat. $122,661-670$

74. Llaverias, G. et al. (2010) A Western-type diet accelerates tumor progression in an autochthonous mouse model of prostate cancer. Am. J. Pathol. 177, 3180-3191

75. Wolfe, A.R. et al. (2015) High-density and very-low-density lipoprotein have opposing roles in regulating tumor-initiating cells and sensitivity to radiation in inflammatory breast cancer. Int. J. Radiat. Oncol. Biol. Phys. 91, 1072-1080

76. Scoles, D.R. et al. (2010) Liver X receptor agonist inhibits proliferation of ovarian carcinoma cells stimulated by oxidized low density lipoprotein. Gynecol. Oncol. 116, 109-116 
708

709

710

711

712

713

714

715

716

717

718

719

720

721

722

723

724

725

726

727

728

729

730

731

732

733

734

735

736

737

738

739

740

77. Gonzalez-Chavarria, I. et al. (2018) LOX-1 activation by oxLDL triggers an epithelial mesenchymal transition and promotes tumorigenic potential in prostate cancer cells. Cancer Lett. 414, 34-43

78. Balzan, S. and Lubrano, V. (2018) LOX-1 receptor: A potential link in atherosclerosis and cancer. Life Sci. 198, 79-86

79. Kuemmerle, N.B. et al. (2011) Lipoprotein lipase links dietary fat to solid tumor cell proliferation. Mol. Cancer Ther. 10, 427-436

80. Balaban, S. et al. (2017) Adipocyte lipolysis links obesity to breast cancer growth: adipocyte-derived fatty acids drive breast cancer cell proliferation and migration. Cancer Metab 5, 1

81. Nieman, K.M. et al. (2013) Adipose tissue and adipocytes support tumorigenesis and metastasis. Biochim. Biophys. Acta 1831, 1533-1541

82. Caro-Maldonado, A. et al. (2018) Low-dose statin treatment increases prostate cancer aggressiveness. Oncotarget. 9, 1494-1504

83. Guillaumond, F. et al. (2015) Cholesterol uptake disruption, in association with chemotherapy, is a promising combined metabolic therapy for pancreatic adenocarcinoma. Proc. Natl. Acad. Sci. U. S. A. 112, 2473-2478

84. Lee, H.J. et al. (2018) Cholesterol Esterification Inhibition Suppresses Prostate Cancer Metastasis by Impairing the Wnt/beta-catenin Pathway. Mol. Cancer Res. 16, 974-985

85. LaPensee, C.R. et al. (2016) ATR-101, a Selective and Potent Inhibitor of Acyl-CoA Acyltransferase 1, Induces Apoptosis in H295R Adrenocortical Cells and in the Adrenal Cortex of Dogs. Endocrinology 157, 1775-1788

86. Li, J. et al. (2016) Abrogating cholesterol esterification suppresses growth and metastasis of pancreatic cancer. Oncogene 35, 6378-6388

87. Lippi, G. et al. (2007) Lipoprotein[a] and cancer: anti-neoplastic effect besides its cardiovascular potency. Cancer Treat. Rev. 33, 427-436

88. Zamanian-Daryoush, M. and DiDonato, J.A. (2015) Apolipoprotein A-I and Cancer. Front Pharmacol. 6, 265

89. Miura, S. et al. (1997) Cholesterol-mediated changes of neutral cholesterol esterase activity in macrophages. Mechanism for mobilization of cholesteryl esters in lipid droplets by HDL. Arterioscler. Thromb. Vasc. Biol. 17, 3033-3040

90. Ruscica, M. et al. (2018) High Density Lipoproteins Inhibit Oxidative Stress-Induced Prostate Cancer Cell Proliferation. Sci. Rep. 8, 2236 
91. Danilo, C. et al. (2013) Scavenger receptor class B type I regulates cellular cholesterol metabolism and cell signaling associated with breast cancer development. Breast Cancer

743 Res. 15, R87

92. Cao, W.M. et al. (2004) A mutant high-density lipoprotein receptor inhibits proliferation of human breast cancer cells. Cancer Res. 64, 1515-1521

93. Ossoli, A. et al. (2019) Dysfunctional HDL as a Therapeutic Target for Atherosclerosis Prevention. Curr. Med. Chem. 26, 1610-1630

94. Pan, B. et al. (2012) High-density lipoprotein of patients with type 2 diabetes mellitus elevates the capability of promoting migration and invasion of breast cancer cells. Int. J. Cancer 131, 70-82

95. Huang, X. et al. (2016) High-density lipoprotein of patients with breast cancer complicated with type 2 diabetes mellitus promotes cancer cells adhesion to vascular endothelium via ICAM-1 and VCAM-1 upregulation. Breast Cancer Res. Treat. 155, 441-455

96. Krause, B.R. and Remaley, A.T. (2013) Reconstituted HDL for the acute treatment of acute coronary syndrome. Curr. Opin. Lipidol. 24, 480-486

97. Morin, E.E. et al. (2018) HDL in Endocrine Carcinomas: Biomarker, Drug Carrier, and Potential Therapeutic. Front Endocrinol. (Lausanne) 9, 715

98. Foit, L. et al. (2015) Synthetic high-density lipoprotein-like nanoparticles for cancer therapy. Expert. Rev. Anticancer Ther. 15, 27-34

99. Tang, J. et al. (2017) Effect of size and pegylation of liposomes and peptide-based synthetic lipoproteins on tumor targeting. Nanomedicine. 13, 1869-1878

100. Mooberry, L.K. et al. (2010) Receptor mediated uptake of paclitaxel from a synthetic high density lipoprotein nanocarrier. J. Drug Target 18, 53-58

101. Zhang, F. et al. (2016) Reconstituted high density lipoprotein mediated targeted codelivery of $\mathrm{HZO}$ and paclitaxel enhances the efficacy of paclitaxel in multidrug-resistant MCF7 breast cancer cells. Eur. J. Pharm. Sci. 92, 11-21

102. Sabnis, N. et al. (2012) Enhanced solubility and functionality of valrubicin (AD-32) against cancer cells upon encapsulation into biocompatible nanoparticles. Int. J. Nanomedicine. 7, 975-983

103. Bray, F. et al. (2018) Global cancer statistics 2018: GLOBOCAN estimates of incidence and mortality worldwide for 36 cancers in 185 countries. CA Cancer J. Clin. 68, 394-424 cancer: highlights of the St. Gallen International Expert Consensus on the Primary Therapy of Early Breast Cancer 2011. Ann. Oncol. 22, 1736-1747 
775

776

777

778

779

780

781

782

783

784

785

786

787

788

789

790

791

792

793

794

795

796

797

798

799

800

801

802

803

804

805

806

807

808

105. Epstein, J.I. et al. (2016) The 2014 International Society of Urological Pathology (ISUP) Consensus Conference on Gleason Grading of Prostatic Carcinoma: Definition of Grading Patterns and Proposal for a New Grading System. Am. J. Surg. Pathol. 40, 244-252

106. Weinberg, S.E. and Chandel, N.S. (2015) Targeting mitochondria metabolism for cancer therapy. Nat. Chem. Biol. 11, 9-15

107. Fischer, K. et al. (2007) Inhibitory effect of tumor cell-derived lactic acid on human T cells. Blood 109, 3812-3819

108. Liu, Y. et al. (2010) Dominant uptake of fatty acid over glucose by prostate cells: a potential new diagnostic and therapeutic approach. Anticancer Res. 30, 369-374

109. Kim, W.Y. (2019) Therapeutic targeting of lipid synthesis metabolism for selective elimination of cancer stem cells. Arch. Pharm. Res. 42, 25-39

110. Havel, R.J. and Kane, J.P. (2001) Structure and metabolism of plasma lipoproteins. In The Metabolic and Molecular Basis of Inherited Diseases (Scriver,C.R., ed), McGraw-Hill

111. Borrelli, M.J. et al. (2019) New Frontiers in Lp(a)-Targeted Therapies. Trends Pharmacol. Sci. 40, 212-225

112. Calabresi, L. et al. (2006) Synthetic high density lipoproteins for the treatment of myocardial ischemia/reperfusion injury. Pharmacol. Ther. 111, 836-854

113. Mundi, S. et al. (2018) Endothelial permeability, LDL deposition, and cardiovascular risk factors-a review. Cardiovasc. Res. 114, 35-52

114. Horton, J.D. et al. (2002) SREBPs: activators of the complete program of cholesterol and fatty acid synthesis in the liver. J. Clin. Invest. 109, 1125-1131

115. Wang, B. and Tontonoz, P. (2018) Liver X receptors in lipid signalling and membrane homeostasis. Nat. Rev. Endocrinol. 14, 452-463

116. Venkateswaran, A. et al. (2000) Control of cellular cholesterol efflux by the nuclear oxysterol receptor LXR alpha. Proc. Natl. Acad. Sci. U. S. A. 97, 12097-12102

117. Zelcer, N. et al. (2009) LXR regulates cholesterol uptake through Idol-dependent ubiquitination of the LDL receptor. Science 325, 100-104

118. Febbraio, M. et al. (2001) CD36: a class B scavenger receptor involved in angiogenesis, atherosclerosis, inflammation, and lipid metabolism. J. Clin. Invest. 108, 785-791

119. Gomaraschi, M. et al. (2006) High-density lipoproteins: a therapeutic target for atherosclerotic cardiovascular disease. Expert. Opin. Ther. Targets. 10, 561-572

120. Vickers, K.C. et al. (2011) MicroRNAs are transported in plasma and delivered to recipient cells by high-density lipoproteins. Nat. Cell Biol. 13, 423-433 


\section{Outstanding questions}

The modulation of plasma levels of lipids by genetic manipulation and/or dietary approaches affected tumor development in mice. However, epidemiological and interventional studies in humans gave inconsistent results. What is the relevance of circulating versus local lipids for tumor development and progression?

The inhibition of cholesterol synthesis and lipoprotein uptake in cancer cells can affect cell viability. To this aim, statins are not promising due to their distribution and mechanism of action. The development of LXR agonists is hampered by their side effects. Other approaches, as the inhibition of the SREBP pathway or the silencing of the LDL-R are under investigation. In addition, the role of lipoprotein remnants in cancer biology has not been addressed to date. What is the optimal target for reducing cholesterol content in cancer cells?

The HDL system seems a promising target to limit cancer cell content of cholesterol and its metabolites. The development of HDL-targeted approaches should consider the role of SR-BI, which could be upregulated in cancer and promote cholesterol uptake from HDL. Could small discoidal reconstituted HDL overcome this problem?

Lipid metabolites as oxysterols and eicosanoids are produced in the TME and can affect both cancer and stromal cells; these metabolites need further dedicated investigations since different molecules seem to exert opposite effects on cancer cells. Is the pattern of oxysterol and eicosanoid production in cancer cell modifiable?

Plasma levels of apoA-I are used as a biomarker for ovarian cancer. Acute reduction of circulating HDL-C along with apoA-I is common during active disease in many cancer types. Could plasma levels of apoA-I be used as a biomarker for other types of tumor? 


\section{Trends}

Tumor microenvironment (TME) can favor tumor progression and TME signature can predict disease outcome. Sources of lipids, pro-inflammatory and pro-oxidant molecules are present in the TME, including lipoproteins.

Cholesterol affects cell proliferation as a component of lipid rafts regulating cell signaling, and as building block for the local synthesis of hormones and oxysterols.

Very low- and low-density lipoproteins in the TME can provide cancer cells with lipids and exert pro-inflammatory and pro-oxidant activities.

High density lipoproteins (HDL) can promote the removal of cholesterol and its metabolites from cancer cell and blunt inflammation and oxidative stress. Boosting the HDL system by the infusion of reconstituted HDL could represent a promising approach to affect cancer cell viability. 